\title{
AMS-3000 LARGE FIELD VIEW AERIAL MAPPING SYSTEM: BASIC PRINCIPLES AND THE WORKFLOW
}

\author{
$\mathrm{Ke} \mathrm{Xi}^{1}$, Yansong Duan ${ }^{1, *}$ \\ ${ }^{1}$ School of Remote Sensing and Information Engineering, Wuhan University, Wuhan 430079, ysduan@whu.edu.cn
}

Commission I, WG I/2

KEY WORDS: Three-line Array Stereo Aerial Camera, Large Field of View, Mapping System, AMS-3000, DPGridAMS

\begin{abstract}
:
Three-line array stereo aerial survey camera is a typical mapping equipment of aerial photogrammetry. As one of the airborne equipment, it can quickly obtain a large range of basic geographic information with high precision. At present, typical three-line array stereoscopic aerial survey cameras, such as Leica ADS40 and 80, have the disadvantages of small field of view and low resolution, which makes it difficult to meet the demand of large-scale topographic mapping for economic construction. For the urgent need of domestic three linear array aerial mapping camera in our project, we developed the AMS-3000 camera system. Camera features include a large field of view, high resolution, low distortion and high environmental adaptability. The AMS-3000 system has reached the international advanced level on both software and hardware aspects.
\end{abstract}

\section{INTRODUCTION}

Three-line array stereo airborne mapping camera can quickly acquire a wide range of high-resolution basic geographic information, which plays a significant supporting role in digital city construction, earthquake relief, resource navigation, environmental protection, homeland security and other works. The airborne digital camera first appeared in the international society for photogrammetry and remote sensing (ISPRS) conference in 2000 (Eckardt et al., 2000). Three-line array stereo airborne mapping camera is an upgrade product of aerial digital camera. Because of its brilliant performance, it has been valued by many countries and has become a research hotspot of aerial photogrammetry (Pechatnikov et al., 2008).

Digital aerial mapping cameras are mainly divided into two categories, one mainly using area-array detectors, the other mainly using linear array detectors (Yao et al., 2018). Both of them have their own advantages and disadvantages (Lin et al., 2019, Zhang et al., 2016). The area-array aerial mapping camera has small pixels and high precision, but its base-height ratio is relatively small, and the elevation accuracy is not as good as that of the linear array aerial survey camera. Moreover, when photographing a large area with high overlap rate, the number of image files is huge, resulting in a long processing time. The linear array aerial camera does not need image splicing. And without a shutter, the linear array camera is more stable to produce more uniform images (Cao et al., 2019). However, the linear array camera is affected by the flight attitude, so a high-precision POS system is necessary (He et al., 2015, Yin et al., 2016).

At present, the well-known stereo airborne mapping cameras include the ADS80, ADS100 of Leica company (linear array detectors), DMC camera of Z/I company and the SWDC camera (area-array detectors) developed by Chinese research institute of surveying and mapping (Boesch et al., 2016). Recent years, the digital aerial mapping camera in China has a preliminary development, but in the degree of automation and accuracy of aerial survey, there is still a significant gap with other high quality cameras. In addition, it is mainly used for area-array aerial mapping cameras, and the technology development is relatively slow. Compared with foreign area-array stereoscopic cameras, such as the DMC of Z/I company, there is still a gap in some key indicators.

Imported cameras are expensive and still have some shortcomings, including difficulties in mapping of small and medium-sized scales, low resolution, small working width and low operating efficiency. These aerial mapping cameras have solved part of the demand of the market to some extent, but they are still far from meeting the demand of China's rapid economic construction for a large amount of aerial remote sensing surveying and mapping geographic information.

Therefore, it is very necessary to develop three-line array stereo airborne mapping cameras with high resolution and large field of view to meet the needs of mapping of large scales (Lu et al., 2016). Based on the above requirements, AMS-3000 camera system was developed.

AMS-3000 large field of view three-line array stereoscopic aerial photography system is a new generation of aerial digital photography system developed by China Academy of Science and Wuhan university. Mounted on a general aviation platform, AMS-3000 can quickly and flexibly acquire high-resolution ground images to achieve 1:1000 large-scale high-precision mapping. The key technology of AMS-3000 is significantly superior to the most advanced ADS40/80 in the world, and it has the high performance to support the economic construction of China.

\section{HARDWARE}

AMS-3000, a three-line array stereo airborne mapping camera with a large field of view, adopts the push-sweep imaging principle. The focal plane of the camera is equipped with multiple high-resolution panchromatic and RGB band line-array detector arrays, which constitute the forward, nadir and backward imaging of the ground. AMS-3000 can

\footnotetext{
* Corresponding author
} 
simultaneously obtain fully overlapping panchromatic PAN band, red single band, green single band and blue single band images, which can directly generate multiple stereo image pairs. At the same time, three-line array stereo airborne mapping camera in large field of view records multi-band images of ground scenes, which can be used for the synthesis of color and false color images.

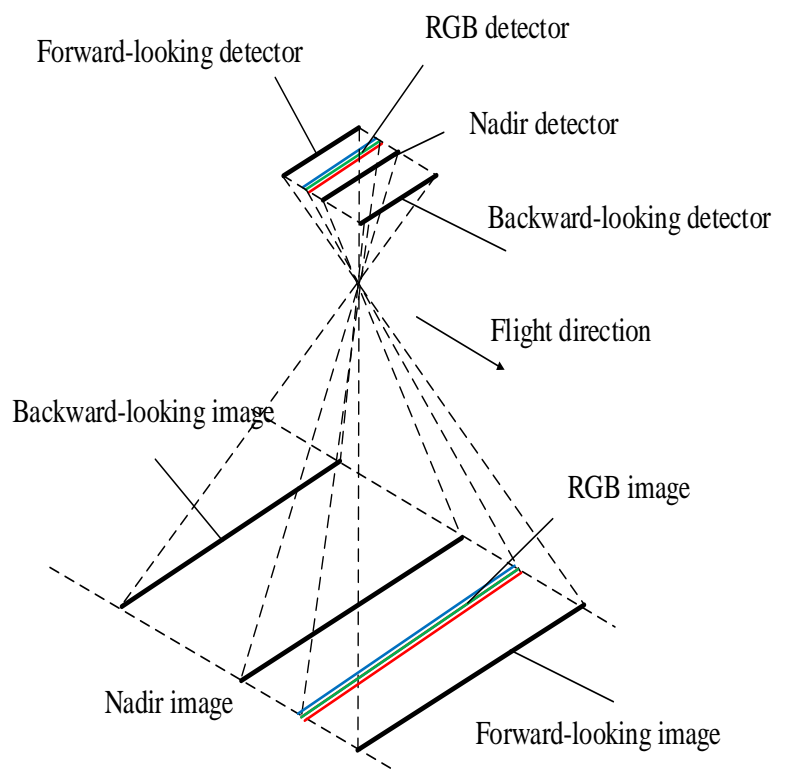

Figure 1. Three-line array camera mapping schematic

As shown in Figure 1, three-line array surveying and mapping camera has three detectors, which are projected on the ground through the optical system. In the process of photogrammetry, if we know the exterior orientation elements (i.e. the position and attitude angle of the camera in the earth coordinate system) and the interior orientation elements (i.e. the main distance, the position of the main point and the intersection angle) of the three-line array cameras at each scanning time, then the image coordinates of any object point on the ground at three different times on the three-line array detectors can be completely determined. On the other hand, if the coordinate of the image point of the corresponding object is calculated, the coordinate of the object point can also be calculated.

The three-line array aerial mapping camera with large field of view adopts a long focal length, single lens transmission optical system and multiple detectors with different angles arranged on the focal plane. Six linear array detectors are mounted on the optical focal plane of the three linear array aerial mapping camera, among which three are panchromatic linear array detectors and the other are R, G and B linear array detectors. These detectors are in parallel with each other and perpendicular to the flight direction. When the camera works, each detector continuously scans the ground in a synchronous period and produces six overlapping strip images, forming panchromatic and multispectral images.

The main technical parameters of AMS-3000 are excellent, which reaches high performance. The AMS-3000 has a volume of $500 \mathrm{~mm} * 500 \mathrm{~mm} * 915 \mathrm{~mm}$ and a weight of $72 \mathrm{~kg}$. Its working temperature is between $-20^{\circ} \mathrm{C}$ to $60^{\circ} \mathrm{C}$. These parameters completely meet common aerial photogrammetry usage scenarios. The camera has a storage capacity of 6TB, which completely meets the data acquisition of large area photographing tasks.
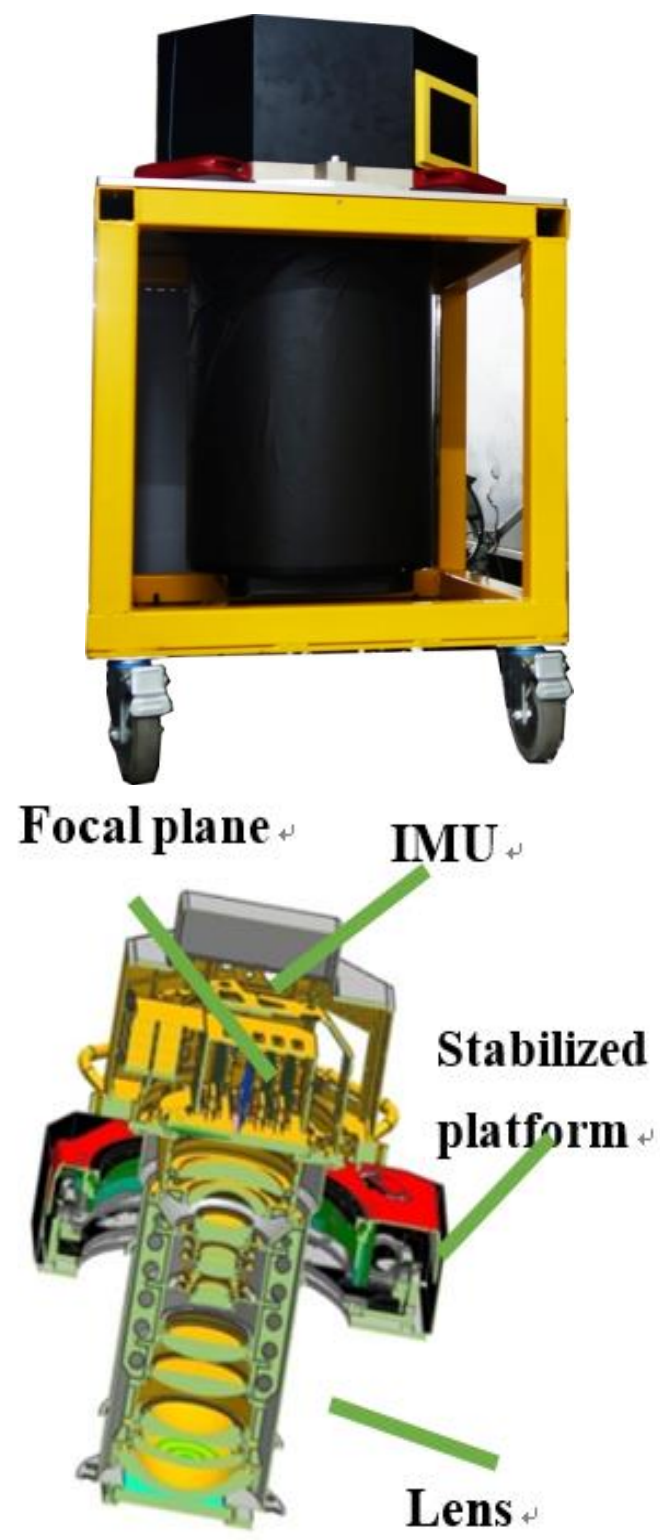

Figure 2. AMS-3000 camera structure

With a scan line width of 32,000 pixels and a base height ratio of 0.89, AMS-3000 supports panchromatic image and RGB color image photographing, which is significantly larger than the mainstream aerial photography cameras. At the flight height of $2400 \mathrm{~m}$, the camera can cover $3000 \mathrm{~m}$ in a single flight band and acquire $0.1 \mathrm{~m}$ resolution remote sensing image of 180 square kilometers in 10 minutes, which has very high data acquisition efficiency. The main features of AMS-3000 camera include:

High resolution panchromatic and RGB images obtained at the same time,

Telecentric lens with high transfer function, lowdistortion and large FOV,

- $\quad$ High working efficiency with 32000 pixels linear array detectors,

- $\quad$ High precision GPS and IMU integrated, ability,

Large base to height ratio and strong stereo imaging - $\quad$ Active temperature control system and airtight design to guarantee environment adaptability,

Automatic data processing system. 
With its unique features, it is designed to meet the needs of worldwide enormous demand with higher image quality, strong stereo imaging ability, high precision GPS and IMU integrated, high working efficiency and reduced costs. AMS-3000 has powerful performance, as shown in Table 1 and Table 2. Compared with the mainstream aerial cameras in the world, its core performance indexes, such as focal length, coverage width $(\mathrm{m})$ at the same resolution $(0.1 \mathrm{~m})$, have exceeded.

\begin{tabular}{|l|c|c|}
\hline Parameters & ADS40/80 & AMS-3000 \\
\hline focal length $(\mathrm{mm})$ & 62.77 & 130 \\
\hline Base to height ratio & 0.78 & 0.89 \\
\hline Pixel number & 12000 & 32768 \\
\hline Pixel size $(\mu \mathrm{m})$ & $6.5 \times 6.5$ & $5 \times 5$ \\
\hline 2000m GSD $(\mathrm{m})$ & 0.207 & 0.077 \\
\hline $\begin{array}{l}\text { Coverage width }(\mathrm{m}) \\
\text { at the same } \\
\text { resolution }(0.1 \mathrm{~m})\end{array}$ & 1206.9 & 3249.3 \\
\hline
\end{tabular}

Table 1 The parameter comparison of ADS40/80 and AMS-3000

\begin{tabular}{|l|c|c|}
\hline Parameters & AMS-3000 & ADS100 \\
\hline focal length & $130 \mathrm{~mm}$ & $62.5 \mathrm{~mm}$ \\
\hline Base to height ratio & 0.89 & 0.78 \\
\hline Intersection Angle & $21^{\circ}, 27^{\circ}$ & $14^{\circ}, 27^{\circ}$ \\
\hline Pixel number & 32768 & 16000 \\
\hline 2000m GSD & $0.08 \mathrm{~m}$ & $0.16 \mathrm{~m}$ \\
\hline $\begin{array}{l}\text { Transverse field of } \\
\text { view }\end{array}$ & $64^{\circ}$ & $60^{\circ}$ \\
\hline $\begin{array}{l}\text { Map scale flight } \\
\text { height 2000m }\end{array}$ & $1: 1000$ & $1: 2000$ \\
\hline Weight & $72.5 \mathrm{~kg}$ & $50 \mathrm{~kg}$ \\
\hline
\end{tabular}

Table 2 The parameter comparison of ADS100 and AMS-3000

\section{SOFTWARE}

The DPGridAMS software was developed by a research team led by Duan Yansong of the school of remote sensing information engineering, Wuhan University. Direct input of the original data obtained by AMS-3000 digital aerial photography, through automatic aerial triangulation, automatic production (DEM), large-scale image color mixing and other process, DEM, DOM, DLG, 3d model data and other mapping products can be produced.

DPGridAMS is a photogrammetric system specially developed by Wuhan University to process image data of domestic three -line array cameras. It is an important part of DPGrid series software of Wuhan University. Consistent with the DPGrid design idea, DPGridAMS is also a distributed processing system based on cluster computers, which can run on either ordinary microcomputer networks or high performance cluster computers (blade servers)

The characteristics and features of DPGridAMS include:

- Based on the distributed network parallel process, and the processing performance is superior,

- Process the original data of the domestic three-line array stereo airborne mapping camera directly,

- Quickly generate T0 level images,

- $\quad$ RGB color image fusion can produce the corresponding T0 level image,

- Semi-automatic control point measurement,

- The block adjustment method is adopted to output the corrected external orientation element,
- $\quad$ DEM is generated based on T0 level image and L1 level image matching,

- Generate orthophoto images

The software can be widely used in basic mapping, urban planning, land resources, satellite remote sensing, military measurement, highway, railway, water conservancy, electric power, environmental protection, agriculture and many other fields of digital city projects.

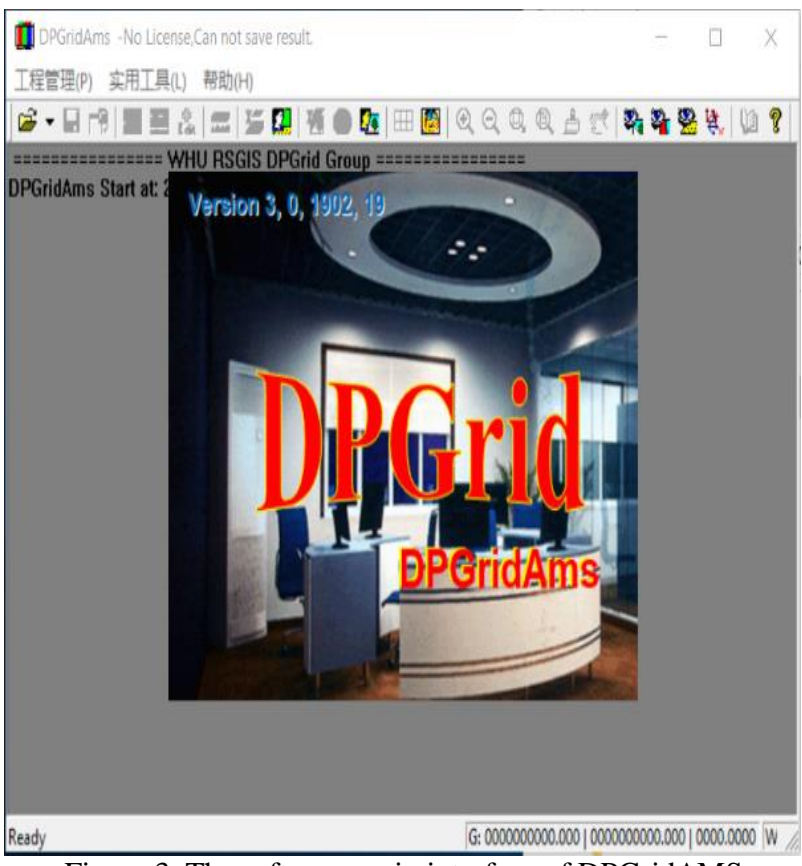

Figure 3. The software main interface of DPGridAMS

With AMS-3000 system, we tested the hardware and software performance of the camera in Yangjiang city, Guangdong province, China. In this production test, the Y-12 type fixed-wing aircraft was used to fly 5 sorties with AMS-3000 digital aerial camera. The effective area obtained was over 1000 square kilometers, and the ground resolution was about 0.1 meter $(2000 \mathrm{~m}$ flight height). The experimental area has many land types, namely, water, residential, forest and coast.

The CCD of three bands of AMS-3000 camera is placed on the focal plane with a distance of 10 microns, and the calibrated panchromatic image is used to automatically calibrate the multi-spectral camera. The calibration parameters of RGB image can be obtained, and the three bands of RGB can be registered to within 2.5 microns through the calibration parameters. The design length of CCD for each row is $163.78 \mathrm{~mm}$, and the calibrated average length is $164.512 \mathrm{~mm}$. The design values of the front and rear CCD viewing angles of the AMS-3000 are 21 degrees and 27 degrees, respectively. The mean viewing angle before calibration is 21.12 degrees, and the mean viewing angle after calibration is 27.21 degrees. The intersection angle during stereo measurement is 48.33 degrees, and the base-to-height ratio of photogrammetry is 0.95 degrees.

The main operating environment for this software is common Windows systems. According to the characteristics of AMS-3000 camera, we refer to the processing flow of ADS80, and redesign the processing flow. The new designed process is more automated and efficient and works well in the AMS-3000 camera. Figure 4 shows the whole advanced workflow of DPGridAMS software. 


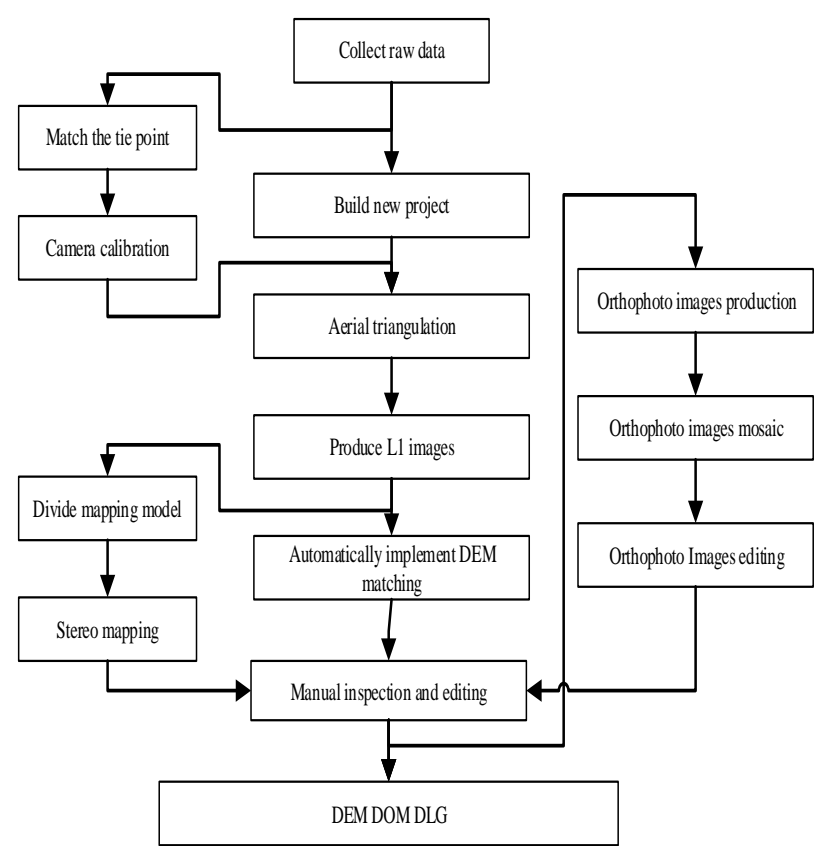

Figure 4. The work flow of DPGridAMS

This system design provides a highly efficient workflow that is able to process large areas that consist of hundreds of thousands of image frames. The improved workflow can better adapt to the original data captured by AMS-3000 camera and improve the efficiency of the whole process. The original camera data, after the whole process above, can get the mapping results of DEM, DOM, and DLG.

The ground processing system of AMS-3000 camera starts from receiving the original data, and carries out radiation calibration and aircraft belt splicing to output level 0 images. Then, it can output level 1 images geometrically according to POS data; Finally, color orthophoto images (level 2 images) with geographic information can be generated according to the processing results of automatic matching and color registration. The data is in standard tiff and geotif formats, and other related parameters are in .odf format and corresponding ellipsoid parameters (XML format), which can be directly provided to users in various industries.

Through aerial photography experiments in different regions, a total of over 100 flight belts were shot, with the image data volume exceeding 20TB. Among these experiments, AMS-3000 system is stable and reliable with excellent performance. Each scanline of the camera has a width of 32,000 pixels. At an altitude of 2,000 meters, the AMS-3000 camera can acquire remote sensing images of 180 square kilometers with a resolution of 0.1 meters every 10 minutes, which has a very high data acquisition efficiency. It has successfully completed the tests in Yangiiang test area of Guangdong Province and Jiamusi test area of Heilongjiang Province in China, and the software and hardware level has reached the expected target.

The test results (Yangjiang test area of Guangdong Province and Jiamusi test area of Heilongiang Province in China) show that the working efficiency and imaging quality of the whole system are excellent, and the accuracy of the images results can meet the requirements of 1:1000 production, which marks the successful development of the first three-line array high-resolution ground data acquisition and processing system in China.
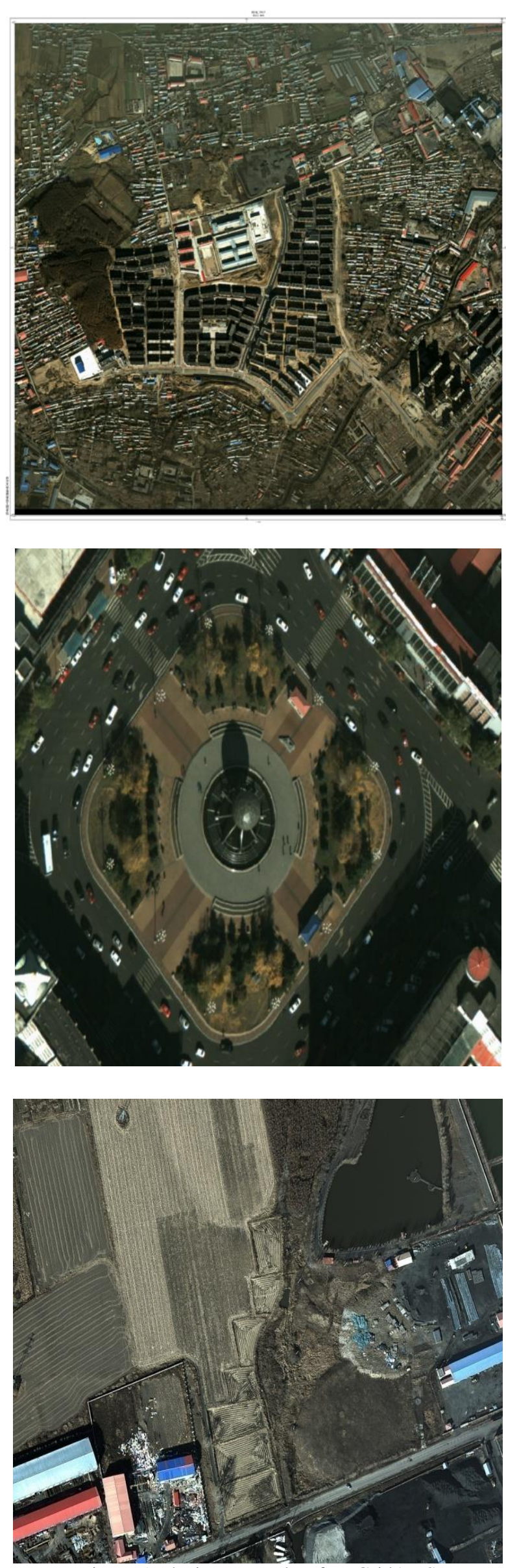

Figure 5. The image results of DPGridAMS 
Figure 5 is part of the result data of AMS-3000 system after flying in Yangjiang test area of Guangdong Province and Jiamusi test area of Heilongjiang Province. Figure 5 shows the orthophoto generated by the DPGridAMS software. It can be seen that the color of the generated orthophoto image is real without color deviation, and the transition of adjacent areas between different images is quite natural without any traces of seam line. Moreover, the radiative resolution of the image is high and the details are very rich. When the image is enlarged, you can clearly see details of vehicles, zebra crossings, trees and buildings on the street.
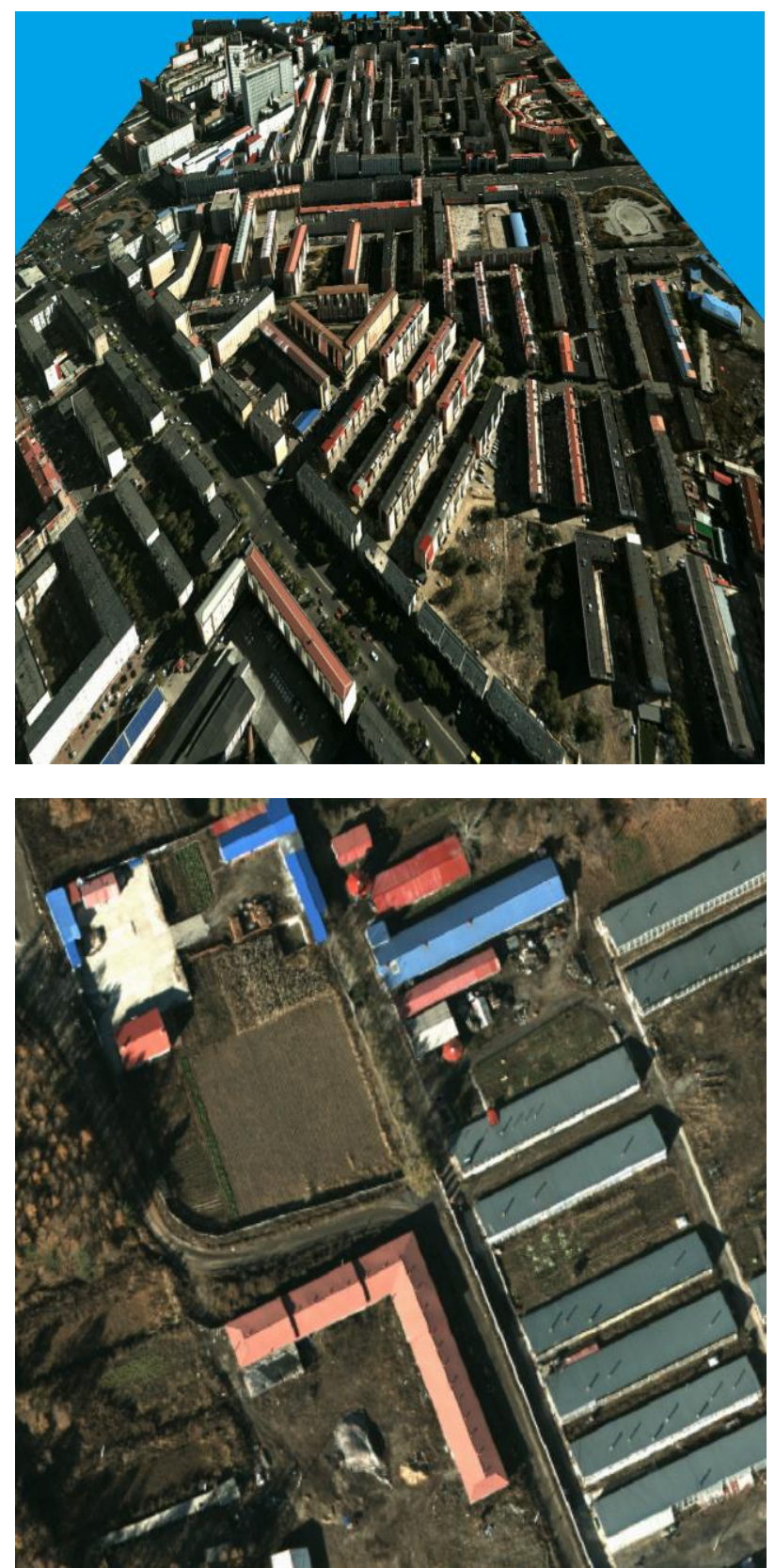

Figure 6. The 3D results of DPGridAMS

Figure 6 shows the 3D models results of DPGridAMS software of Yangjiang test area. Because AMS-3000 camera has high resolution and accurate positioning, the 3D models produced from these images are also exquisite. The entire model has a large enough scope that the details of each building are fully presented. And there is no evident dislocation in the large 3D model.

\section{CONCLUSION}

This paper introduces a new generation of three-line array stereo airborne mapping camera AMS-3000 and the supporting software processing system DPGridAMS. The system adopts a new optical design to solve the restriction between large field of view and high resolution. The most important features are:

- $\quad$ Large field view,

- High resolution,

- Low distortion,

- Large FOV,

- High precision GPS and IMU integrated,

- Hardware and software integration,

- Distributed software operating system with high processing efficiency,

- High degree of software automation.

All the above features of the AMS - 3000 have been implemented and proven by test and calibration facilities. In the future, the AMS-3000 system will be updated continuously to meet the growing domestic demand.

\section{REFERENCES}

Pechatnikov, M., Shor, E., Raizman, Y., 2008. VisionMap A3 super wide angle mapping system basic principles and workflow. International Archives of the Photogrammetry, Remote Sensing and Spatial Information Sciences. Vol. XXXVII, pp. 1735-1740.

Eckardt, A., Braunecker, B., Sandau, R., 2000. Performance of the imaging system in the LH Systems ADS40 airborne digital sensor, International Archives of Photogrammetry and Remote Sensing , XXXIII(B), pp. 104-109.

Yao, Y., Xu, Y., Ding Y., Yuan G., 2018. Optical-system design for large field-of-view three-line array airborne mapping camera. Optics and Precision Engineering , 26(9): 2334-234

He, Y., Xiao, X., Li, Y., Shao, X., Wang, N., Qiang H., Lu F., 2015. Optical system design for the aerial camera with dual band, common optical path, long focal, oblique view and focusing, AOPC 2015: Optical Test, Measurement, \& Equipment. International Society for Optics and Photonics, vol. 9677, p. 96771O. doi:10.1117/12.2199775

Boesch, R., Bühler, Y., Marty, M., Ginzler, C., 2016. Comparison of digital surface models for snow depth mapping with UAV and aerial cameras. International Archives of the Photogrammetry, Remote Sensing and Spatial Information Sciences. Volume XLI-B8, pp. 453-458.

Yin, L., Wang, X., Ni, Y., Zhou, K., Zhang, J., 2018. Extrinsic parameters calibration method of cameras with non-overlapping fields of view in airborne remote sensing. RemoteSensing,10(8):1298. doi:10.3390/rs10081298

Cao, G., Liu, X., Zhu, D., 2019. Novel optical design of the large format aerial measuring camera with large field and low distortion, AOPC 2019: Space Optics, Telescopes, and Instrumentation. International Society for Optics and Photonics, 11341: 113411Z. doi:10.1117/12.2550691

Zhang, Z., Rebecq, H., Forster, C., Scaramuzza, D., 2016. Benefit of large field-of-view cameras for visual odometry, 2016 IEEE International Conference on Robotics and Automation (ICRA). IEEE:801-808 
Lu, Y., Wang, K., Fan, G., 2016. Photometric calibration and image stitching for a large field of view multi-camera system. Sensors, 16(4): 516. doi:10.3390/s16040516

Lin, J., Jiang, C., Lu, M., Gao, M., Guo, Q., 2019. Development of an aspherical aerial camera optical system. IEEE Photonics Journal, vol. 11, no. 6, pp. 1-13. 